\title{
Force Feedback Magnitude Effects on User's Performance during Target Acquisition: A Pilot Study
}

\author{
Lode Vanacken, Joan De Boeck, and Karin Coninx \\ Hasselt University - tUL - IBBT, Expertise Centre for Digital Media (EDM) \\ Wetenschapspark 2 B-3590 Diepenbeek (Belgium) \\ \{lode.vanacken, joan. deboeck, karin. coninx\} @uhasselt. be
}

\begin{abstract}
Only a few guidelines exist for defining the force properties in a haptic interface; as a consequence, they are mostly determined in an ad-hoc manner. We investigate how the user's performance changes during target acquisition when increasing force amplitudes are applied. Using a simple multidirectional point-select task, forces with variable amplitudes are applied to the user while traversing from one target to the other. We find that the user's performance suddenly degrades significantly, rather than decreasing progressively. This finding may be important for defining guidelines which forces may and may not be applied to a user in order to allow the haptic feedback to improve, rather than deteriorate the user's performance.
\end{abstract}

Keywords: Force Feedback, Target Acquisition.

\section{Introduction and Related Work}

Force feedback can be applied to support pointing tasks in a desktop application or in a virtual environment. This can be achieved either by assisting the user e.g. using 'gravity wells' (a 'snap-to' effect that pulls the user's pointer to the centre of the target) [1] or by giving haptic feedback in the form of a bump or a vibration as soon as an event occurs or a given zone is entered [2]. As not many guidelines exist, finding suitable values for the different parameters that define the forces is mostly performed using a 'trial-and-error' approach.

In our research, we want to answer the fundamental question what kind of forces may or may not be applied in order to support the user. Ultimately, this approach may lead to a set of guidelines avoiding the aforementioned trial-and-error approach. In this paper we want to investigate the user's behaviour with respect to the task completion time with changing forces. However, 'changing' a force may influence a number of degrees of freedom: force feedback device, force shape, duration, amplitude, force direction, ...). Within the scope of this paper we define the force as a short force in a given direction, with given duration and amplitude. The amplitude of the force over time may follow a mathematical pattern such as a sine or a step function, which we define as the force shape. Fig. 1a illustrates such a sinusoidal 'force bump' which may occur lateral or longitudinal with respect to the user's movement. 

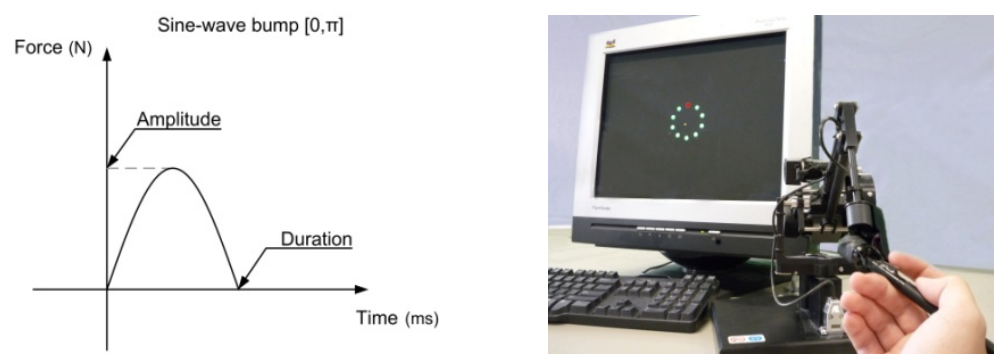

Fig. 1. a. (left) Force evolution of a sinusoidal haptic bump. b. (right) Setup of the experiment.

\section{Experiment}

We conducted a multi-directional tapping experiment in which we evaluate the influence of the force magnitude on the performance of the user.

\subsection{Setup}

As can be seen from Fig. 1b, our experimental setup consisted of a regular 19-inch CRT monitor and a Phantom premium 1.0 haptic device. The control display gain was 1 (one physical $\mathrm{cm}$ corresponds to one $\mathrm{cm}$ on the screen). Ten participants (one female and nine males) with an age between 22 and 30 (average 25), recruited among our co-workers, participated. All participants were right-handed and used their dominant hand during the experiment.

A simple multidirectional point-select task, as described in ISO 9241-9 [3], was used for this experiment. Ten targets are placed in a circle on the screen (see Fig. 1b). The diameter of the circle is determined at $6 \mathrm{~cm}$ and the size of a target is $0.7 \mathrm{~cm}$. This task has a Fitts' index of difficulty of 3.26 bits, a measure typically used in Fitts' law experiments to indicate the difficulty of the task [4]. The value is chosen to be comparable to the task difficulty of a typical icon selection task [5].

During the test, the ten targets were highlighted one after the other and users were requested to select (by pointing and clicking) the highlighted target as fast and accurate as possible. Highlighting is altered between opposite sides of the circle so that user movements are equally distributed among all directions with a maximum distance between the targets. As the task to perform was a $2 \mathrm{D}$ selection task and the haptic device we used is a 3D input device, a vertical guiding plane restricted the task to two dimensions.

Finally, force feedback appearing in the form of a force bump with given shape, duration and varying amplitude was activated when exactly half-way in the path to the next target.

\subsection{Design}

A repeated measures within-participant design was adopted. The independent variables were force direction $D$ (lateral to movement direction $\mathrm{d}_{\text {lat }}$, longitudinal in the direction of the movement $\mathrm{d}^{+}$long and longitudinal opposed to the movement $\mathrm{d}_{\text {long }}$ ) and force amplitude $A$ (see Fig. 2). A fully crossed design resulted in 33 combinations of 

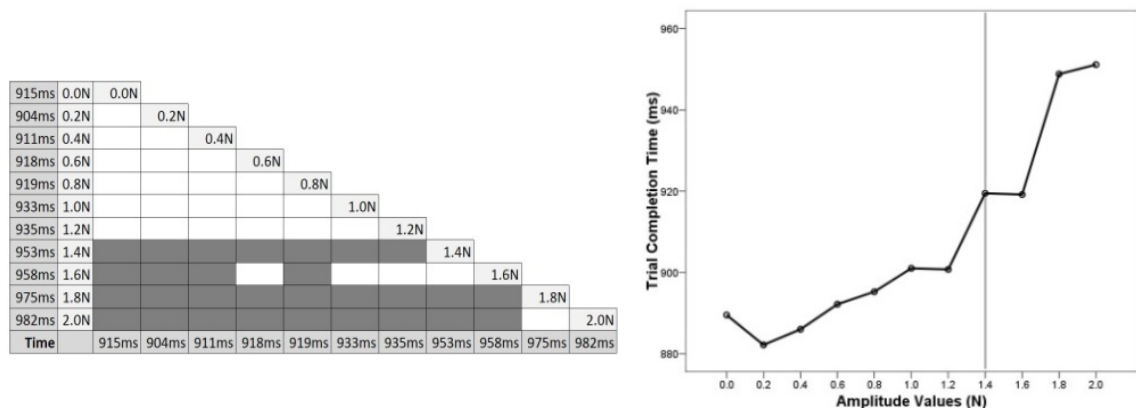

Fig. 2. a. (left) Pair wise post hoc comparisons of the amplitude conditions for trial completion time, all significant differences $(\mathrm{p}<.05)$ are shaded. $b$. (right) Trial completion times for the different amplitudes.

$A$ and $D$. Each participant performed the experiment in one session lasting about 20 minutes. The session consisted of 5 blocks with each block containing the 33 combinations ( $11 \mathrm{As}$ and $3 \mathrm{Ds}$ ) repeated 3 times in a random order. For a total of 99 trials per block, this resulted in 495 trials per participant. Between each block, users were obliged to take a break of at least 15 seconds to minimise fatigue during the test. Before the experiment, participants were given all 33 conditions in random order to familiarise them with the task. During the experiment the time it took to select a target was recorded, as well as the amount of errors made during selection.

\subsection{Results and Discussion}

We want to eliminate the results of any learning effects in our analysis; therefore we first investigated the influence of the block on the trial completion time. From this analysis, blocks 1 and 2 were significantly slower and were hence removed $\left(\mathrm{F}_{4,36}=\right.$ $5.3, \mathrm{p}<.003)$.

Trial Completion Time. A repeated measures analysis of variance of the faultless trials, showed main effects for $D\left(\mathrm{~F}_{2,18}=5.42, \mathrm{p}<.02\right)$ and $A\left(\mathrm{~F}_{10,90}=12.6\right.$, $\mathrm{p}<.0001)$. The average trial completion times for direction are $901.5 \mathrm{~ms}$ for $\mathrm{d}_{\text {long, }}^{+}$, $916.2 \mathrm{~ms} \mathrm{~d}_{\text {long }}$ and $905.5 \mathrm{~ms}$ for $\mathrm{d}_{\text {lat }}$. The differences between the $D$-conditions are small with respect to practical use. Post hoc comparison showed no significant differences except between $\mathrm{d}^{+}$long and $\mathrm{d}_{\text {long. }}^{-}$This may not be surprising, as the first is assisting the user's movement while the latter is opposing it.

Post hoc comparisons for $A$ showed many significant results. Fig. 2a represents these differences $(\mathrm{p}<.05)$ darkly shaded. Comparing the no-force condition with all other $A$-s (first column), a significant trial completion time deterioration, all $\mathrm{p}<.002$, can be seen from $A=1.4 \mathrm{~N}$. Fig. 2a shows that this deterioration is also confirmed by the comparison of the other $A$ conditions. Fig. $2 b$ also depicts all the average completion times for all $A$ values: we see a small but non-significant deterioration for the smaller amplitudes and a stronger, significant degradation starting from 1.4N. This cut-off behaviour may be interesting for further research, when trying to formulate guidelines which forces may and may not support the user's task. 
Error Rates. The same pattern can be found for the error-rate. An error was recorded when the user clicked the button without having the correct target underneath the cursor. The overall error rate for the experiment was 114 errors or $2.3 \%$. The direction had no significant effect on error rate $D\left(\mathrm{~F}_{2,18}=.668, \mathrm{p}=.525\right)$. However, the amplitude values did show a significant effect $A\left(\mathrm{~F}_{10,90}=2.62, \mathrm{p}<.01\right)$. Post hoc comparisons showed that the no-force condition $(0.7 \%)$ has a significant difference with the last two $A$ conditions ( $1.8 \mathrm{~N}$ and $2.0 \mathrm{~N}$, respectively $5.2 \%$ and $5.6 \%$ ). In the scope of this finding, we might refer to the remarks of several users reporting involuntary miss-clicks due to the large forces, and due to the forces diverting them from an anticipated target click.

\section{Conclusion and Future Work}

We investigated the user's performance when forces with different amplitudes are applied during a targeting task. We observed performance deterioration as forces become stronger. It is important to notice that the degradation occurs according to a cut-off behaviour, rather than decreasing progressively. This pilot study only took the force amplitude and direction into account. However, several other parameters such as duration and shape can be changed as well. From the physical relation between force, acceleration and velocity, we foresee that the calculation of the integral may be a good prediction of the user's behaviour, which we will investigate in our future work.

\section{References}

1. Hwang, F., Langdon, P., Keates, S., Clarkson, J.: The effect of multiple haptic distractors on the performance of motion-impaired users. In: Eurohaptics 2003, pp. 14-25 (2003)

2. Forlines, C., Balakrishnan, R.: Evaluating tactile feedback and direct vs. indirect stylus input in pointing and crossing selection tasks. In: CHI 2008, pp. 1563-1572 (2008)

3. ISO, International Organisation for Standardisation. ISO/TC 159/SC4/WG3 N147. Ergonomic requirements for office work with visual display terminals (VDTs) - Part 9 - Requirements for nonkeyboard input devices, May 25 (1998)

4. Fitts, P.: The information capacity of the human motor system in controlling the amplitude of movement. Journal of Experimental Psychology 47, 381-391 (1954)

5. Soukoreff, R.W., MacKenzie, I.S.: Towards a standard for pointing device evaluation, perspectives on 27 years of fitts' law research in HCI. Int. J. Hum.-Comput. Stud. 61(6), 751789 (2004) 